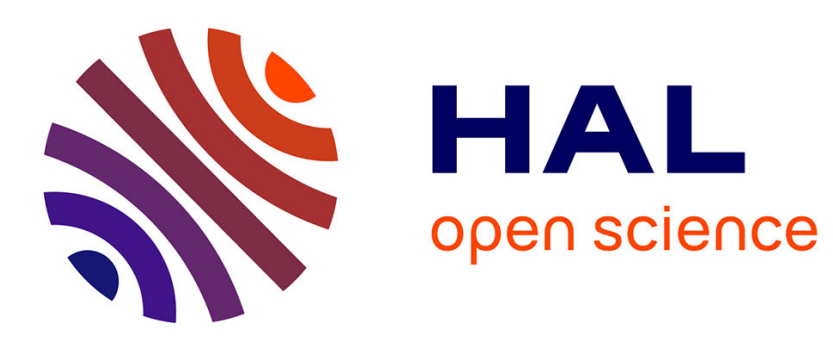

\title{
Highly Anisotropic Rigidity of "Ribbon-Like" Polymers: III. Phase Diagrams for Solutions
}

Irina Nyrkova, Alexander Semenov, Jean-François Joanny

\section{To cite this version:}

Irina Nyrkova, Alexander Semenov, Jean-François Joanny. Highly Anisotropic Rigidity of "RibbonLike" Polymers: III. Phase Diagrams for Solutions. Journal de Physique II, 1997, 7 (6), pp.847-875. 10.1051/jp2:1997159 . jpa-00248482

\section{HAL Id: jpa-00248482 https://hal.science/jpa-00248482}

Submitted on 1 Jan 1997

HAL is a multi-disciplinary open access archive for the deposit and dissemination of scientific research documents, whether they are published or not. The documents may come from teaching and research institutions in France or abroad, or from public or private research centers.
L'archive ouverte pluridisciplinaire HAL, est destinée au dépôt et à la diffusion de documents scientifiques de niveau recherche, publiés ou non, émanant des établissements d'enseignement et de recherche français ou étrangers, des laboratoires publics ou privés. 


\title{
Highly Anisotropic Rigidity of "Ribbon-Like" Polymers: III. Phase Diagrams for Solutions
}

Irina A. Nyrkova $\left({ }^{1,2}\right)$, Alexander N. Semenov $(1,2,3,4, *)$ and Jean-François Joanny $\left({ }^{2}\right)$

( ${ }^{1}$ Physics Department, Moscow State University, 117234 Moscow, Russia

$\left({ }^{2}\right)$ Institut Charles Sadron, 6 rue Boussingault, 67083 Strasbourg Cedex, France

( ${ }^{3}$ ) Department of Applied Mathematics, University of Leeds, Leeds LS2 9JT, UK

$\left({ }^{4}\right)$ Nesmeyanov Institute of Organo-Element Compounds of Russian Academy of Science, 28 Vavilova Str., 117812 Moscow, Russia

(Received 25 October 1996, accepted 28 February 1997)

PACS.61.25. Hq - Macromolecular and polymer solutions, polymer melts; swelling

PACS.61.30.-v - Liquid crystals

PACS.64.70.-p - Specific phase transitions

\begin{abstract}
The behavior of solutions of polymer chains with anisotropic rigidity is considered theoretically. The diagrams of different scaling regimes and the phase diagrams for polymers with arbitrary contour length $L$, inplane rigidity $l$, and out of plane rigidity $l_{2}$ are obtained. Two new types of nematic structures in addition to ordinary nematic with ordering of tangent vectors are found for the regime of moderate second rigidity $l_{2}\left(l \lesssim l_{2} \lesssim l^{3} / d^{2}\right)$ : a pancake nematic (with ordering of the normals to the planes of main flexibility of the chains) and a biaxial nematic (when both tangent and normal vectors are ordered). We also found smectic $A$ and smectic $C$ phases in addition to three nematic phases. The scaling (modified blob) and mean-field with correlation corrections approaches are developed and used when appropriate. Order parameters, correlation radii and chain sizes are discussed for most of the regimes.
\end{abstract}

\section{Introduction}

Polymers with anisotropic rigidity have become available quite recently. These are either soluble ladder polymers [1,2] or living polymers formed by the aggregation of peptide rods [3]. These polymers have specific ribbon-like (or tape-like) structure and possess unusual rigidity: they rather freely bend inside one plane (the plane of main flexibility, PMF), but their out of plane rigidity is extremely high. In the two previous papers of this series, we have considered the conformation of an isolated chain with planar rigidity and the various possible symmetries of the liquid crystalline ordering that is expected in more concentrated solutions $[4,5]$. More specifically, in the second paper [5], we have constructed a general approach to describe nematic ordering in solutions of ribbon polymers. These two papers [4] and [5] will be referred to below as paper I and paper II respectively; the references to the sections and equations of these papers are denoted below as "I.x.y" (or "II.x.y") where "x.y" is the number from papers I or

(*) Author for correspondence (e-mäil: A.Semenov@leeds.ac.uk)

(C) Les Éditions de Physique 1997 
II. The list of symbols used throughout these three papers (together with the present one) is presented at the end of this paper.

In paper I we have justified a persistent-like model for the statistics of ribbon polymers: the orientational correlations of the tangent vectors $\mathbf{t}$ and of the vectors $\mathbf{n}$ normal to the PMF decay exponentially if the chemical distance between two points is large enough (the corresponding persistence lengths are $l$ or $l_{2}$, where $l_{2} \gtrsim l$ is the second persistence length corresponding to the "out of plane" rigidity (see Eqs. (I.2.2, I.2.4))). We have investigated the characteristic shape of isolated chains (in the limit of extremely low concentrations) and shown that the chain behavior essentially depends on the dimensionless parameter

$$
\Omega \equiv l_{2} d^{2} / l^{3}
$$

where $d$ is the characteristic chain thickness. We have shown that the anisotropy in the rigidity induces a shape anisotropy of isolated chains, so that their fragments tend to adopt a disc-like conformation. For large $\Omega(\Omega \gtrsim 1)$ relatively short chain fragments (with contour lengths $s$ : $l \lesssim s \lesssim l \Omega^{2 / 5}$ ) look like absolutely flat coins (with a perpendicular size $R_{\perp} \simeq d$ ), for larger scales $\left(l \Omega^{2 / 5} \lesssim s \lesssim l_{2}\right)$ the chain adopts a thick disc conformation (when $R_{\perp} \gtrsim d$, but the coils are still anisotropic: $R_{\|} \gg R_{\perp}$ ) (see Eq. (I.3.11)). For smaller values of $\Omega\left(d^{2} / l^{2} \lesssim \Omega \lesssim 1\right.$, or $l \lesssim l_{2} \lesssim l^{3} / d^{2}$ ) the flat coin regime disappears, but the chains are still anisotropic (disc-like) up to a contour length $s \sim l_{2}$ (Eq. (I.3.10)). For $l_{2} \sim l\left(\Omega \sim d^{2} / l^{2}\right)$ the anisotropy of the rigidity is not important, and the chains behave as standard semiflexible chains with an isotropic rigidity.

Once the behavior of isolated ribbon chains is understood, it is natural to proceed to consider the case of more concentrated solutions using the standard scaling approach, as done for example in reference [6]. However, the problem is complicated by the existence of various possible orientational transitions which are related to the shape anisotropy of the coils. As discussed in paper $\mathrm{I}$, one can expect several types of nematics phases in solutions of tape-like polymers (which we called $\mathrm{P}-, \mathrm{N}$ - and $\mathrm{B}$-nematics), and also smectic $\mathrm{A}$ and smectic $\mathrm{C}$ phases. The origin of these phases can be explained as follows.

It is well-known that lyotropic nematic crystals are formed in rather concentrated solutions of semirigid polymers. Ordinary persistent chains form nematic liquid crystalline phases ( $N$-phase) where the main axes of the chains (tangent vectors $\mathbf{t}$ ) tend to lay parallel to each other [7-9]. Disc-like objects also tend to order in a liquid crystalline phase where the disc planes (or the PMFs for the case of ribbon polymers) tend to have a parallel orientation $[7,10-12]$, we called this structure a pancake $(P-)$ nematic. When both $\mathbf{t}$-vectors and $\mathbf{n}$-vectors are ordered (in perpendicular directions) a biaxial nematic ( $B$-nematic) phase is formed. For detailed explanations about the $\mathrm{P}, \mathrm{N}$ and $\mathrm{B}$ phases, we refer the reader to paper I (Sect. I.4.1).

When the second rigidity is high $(\Omega \gtrsim 1)$ the chain fragments adopt a flat coins conformation of standard width $d$. For lower $\Omega\left(l \lesssim l_{2} \lesssim l^{3} / d^{2}\right)$ short fragments of contour length $x \lesssim\left(d^{2} l_{2}\right)^{1 / 3}$ also are anisotropic and have standard width $d$. Hence the possibility of formation of smectic phases with periodicity $\sim d$ (where the coins are arranged in layers of width $\sim d$ ) must be considered (as in Sect. I.4.2). If the orientation of the tangent vectors $\mathbf{t}$ inside the layers is random, a smectic-A phase $\left(S_{A}-\right.$ phase) is formed. At higher concentrations, the tangent $\mathbf{t}$-vectors tend to have a parallel alignment inside each smectic layer, thus forming a smectic $\mathrm{C}\left(S_{C}\right)$ phase.

The aim of the present paper is to obtain a complete set of diagrams showing the different regimes and phases of solutions of chains with two rigidities. Our strategy is the following. For any given set of parameters $\left\{d, l, l_{2}, L, \Phi\right\}$ which characterize the solution we perform a 
scaling analysis and determine the most stable liquid crystalline phase. In each phase, we find the correlation lengths and the average chain sizes. The diagrams are presented in a plane concentration vs. contour length $(\{\Phi, L\})$, for given values of $\Omega(1.1)$.

In the next section we discuss the free energy of the orientationally ordered system. The phase diagram in the case of extremely low second rigidity $\left(l_{2} \sim l\right)$, when the properties of the system are undistinguishable from those of ordinary persistent chain solutions $\left(l_{2}=0\right)$, is considered in Section 3. Although these systems are well understood, we need to reformulate the known results using our variables $\{\Phi, L\}$. We also discuss the scaling results on the correlation properties in the isotropic phase (see Refs. [6,14-16]) and in the orientationally ordered phase [17].

In following sections we proceed to study higher rigidities, along the lines of the diagrams of Figure I.3(a-c) for the single chain properties. Section 4 deals with the case $l \lesssim l_{2} \lesssim l(l / d)^{4 / 3}$, Section 5 with $l(l / d)^{4 / 3} \lesssim l_{2} \lesssim l^{3} / d^{2}$, and finally Section 6 with $l_{2} \gtrsim l^{3} / d^{2}$ (i.e. $\Omega \gtrsim 1$ ).

\section{Free Energy}

The theoretical approaches treating uniaxial nematic phases in solutions of ribbon polymers were discussed in paper II. We showed that the free energy (per chain) in the P-phase can be written as (see Eqs. (II.3.5, II.5.7)):

$$
\begin{gathered}
\mathcal{F} \sim \ln \Phi+\ln \frac{1}{\Psi}+\frac{L}{l_{2}} \Psi^{-2}+\Phi \frac{L}{d} b(\Psi) /(1+\mathcal{N}) ; \\
b(\Psi) \simeq 1+\Psi^{2}
\end{gathered}
$$

where $\Phi$ is the volume fraction occupied by the chains, $L$ is the chain contour length, $\Psi=\sin \psi$, $\psi$ is the orientation angle that measures the width of the orientational distribution of the $\mathbf{n}$ vectors, and $\mathcal{N}$ is the average number of secondary contacts coating any primary contact between two chain fragments (interaction blobs). Note that we omit numerical coefficients of order unity which are not important for our scaling analysis; we also use $k_{\mathrm{B}} T$ as the energy unit.

A biaxial nematic can be imagined as a system of nearly parallel disk-like coils with an orientation ordering inside each disk along some axis parallel to the disks (say, the $z$-axis). This ordering can be described by the orientation angle $\gamma$ measuring the width of the orientation distribution of tangential vectors. The distribution of $\mathbf{n}$ vectors (which are preferably oriented along $x$-axis) in a biaxial nematic is characterized by two orientation angles $\psi_{1}$ and $\psi_{2}$ corresponding to a rotation around the $z$-axis and around the $y$-axis respectively. The generalization of the free energy expression (Eq. (2.1)) to the biaxial case is straightforward. Using the approach detailed in paper II we get here

$$
\mathcal{F} \sim \ln \Phi+\ln \frac{1}{\Gamma \Psi_{1} \Psi_{2}}+\frac{L}{l} \Gamma^{-2}+\frac{L}{l_{2}}\left(\Psi_{1}^{-2}+\Psi_{2}^{-2}\right)+\Phi \frac{L}{d} \Gamma b\left(\Gamma, \Psi_{1}, \Psi_{2}\right) /(1+\mathcal{N})
$$

where

$$
b\left(\Gamma, \Psi_{1}, \Psi_{2}\right)=1+\Psi_{1}^{2}+\Psi_{2}^{2} / \Gamma^{2}
$$

and $\Gamma=\sin \gamma, \Psi_{1}=\sin \psi_{1}, \Psi_{2}=\sin \psi_{2}$ are the order parameters in the $\mathrm{B}$ phase. Here the first term corresponds to the ideal-gas translational entropy, the next three terms account for the orientational entropy, and the last term is the excluded volume interaction. 
Note that a uniaxial nematic with ordering of the $\mathbf{t}$ vectors only ( $\mathrm{N}$-phase) corresponds to $\Psi_{1}=1, \Psi_{2}=\Gamma$, and thus the free energy for the uniaxial case reduces to

$$
\mathcal{F} \sim \ln \Phi+2 \ln \frac{1}{\Gamma}+\frac{L}{l} \Gamma^{-2}+\Phi \frac{L}{d} \Gamma
$$

in agreement with equation (II.4.8) (here we also take into account that for a uniaxial nematic $\mathcal{N}$ is always negligible, as was shown in Sect. II.4.2).

A complete analysis of the orientation behavior of solutions of chains with two rigidities is possible on the basis of equations (2.2): at any given $\Phi$ one can minimize the free energy (2.2) vs. order parameters $\Gamma$ and $\Psi_{1}, \Psi_{2}$, and then get the phase diagram using standard techniques (see, e.g. Refs. $[13,14])$. However, in order to proceed, we need to define the number of secondary contacts $\mathcal{N}$ for all phases. For a pancake nematic phase $\mathcal{N}$ was calculated in paper II:

$$
\begin{aligned}
& \mathcal{N} \simeq \frac{\tilde{\Lambda}^{1 / 2} d}{l^{3 / 2} \Psi}, \quad l<l_{2}<l^{3} / d^{2} \\
& \mathcal{N} \simeq \frac{1}{\Psi}\left(\frac{\tilde{\Lambda}}{L_{6}}\right)^{1 / 2}+\varphi\left(\frac{d}{l \Psi}\right), \quad l_{2}>l^{3} / d^{2}
\end{aligned}
$$

where $\varphi(x) \simeq x /\left(1+x^{1 / 3}\right)$, and $L_{6}=l_{2} \Omega^{-2 / 5}$ (see Eqs. (II.4.14, II.4.17)). Equation (2.4) are valid in the range $l<\tilde{\Lambda}<\Psi^{2} l_{2}$. The cut-off length $\tilde{\Lambda}$ is the contour length $\Lambda$ of an interaction blob which is defined by the condition that the interaction energy per interaction blob is of order $k_{\mathrm{B}} T$. In the case where the interaction energy of the whole chain is smaller than $k_{\mathrm{B}} T$ (e.g. in a dilute solution), the cutoff length $\tilde{\Lambda}$ is equal to the chain length $L$. Thus $\tilde{\Lambda}=L$ in the dilute and $\Theta$ regions, and in general case $\tilde{\Lambda}=\min (\Lambda, L)$. In the mean-field region, where the correlation correction is small $(\mathcal{N} \ll 1)$, the interaction energy per chain fragment $\Lambda$ is $F_{\text {int }} \sim \frac{\Lambda}{d} \Phi \Gamma ;$ therefore

$$
\Lambda \sim \frac{d}{\Phi \Gamma}, \quad \tilde{\Lambda}=\min \left(L, \frac{d}{\Phi \Gamma}\right) .
$$

When $\mathcal{N} \gg 1$ the interactions are strong and the solution properties can be obtained from a standard scaling approach, where the interaction blob is equal to the concentration blob.

\section{Phase Diagram of Polymers with a Vanishing Second Rigidity}

We first discuss the case where the effect of the second rigidity is negligible: $\Omega \lesssim(d / l)^{2}, l_{2} \lesssim l$. In this case the chain conformation is either a rod or an isotropic coil and the phase diagram coincides with that of ordinary semiflexible chains. The characteristic sizes of the coils are defined in equation (I.3.9) (see Fig. I.3a):

$$
R_{0} \sim\left\{\begin{array}{ccc}
L & \text { if } & d \lesssim L \lesssim l \\
(L l)^{1 / 2} & \text { if } & l \lesssim L \lesssim L_{4} \equiv l^{3} / d^{2} \\
L^{3 / 5}(d l)^{1 / 5} & \text { if } & L \gtrsim L_{4}
\end{array}\right.
$$

The corresponding diagram of regimes is shown in Figure 1. Below we just recall briefly the main features of the system in order to demonstrate the scaling approach and to set up the reference point for further comparison with true ribbon-like polymers considered in Sects. 4-6. 


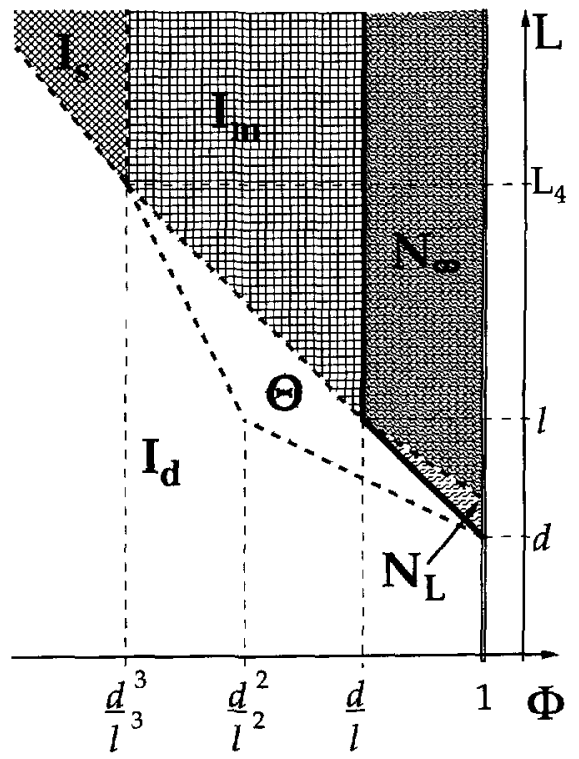

Fig. 1. - Phase diagram for solutions of ribbon-like polymers in variables $\Phi$ (polymer volume fraction), $L$ (polymer contour length) for the case of a vanishing second rigidity: $l_{2}=0$. Thick solid lines correspond to the first order transitions; dashed lines are the cross-over lines. Isotropic solutions are denoted as $I$ or $\Theta$; nematic phase as $N$.

3.1. ISOTROPIC PHASE. - At low concentrations the system is isotropic and its properties can be analyzed via standard scaling analysis $[6,15,18]$. At very low concentrations (in the region $I_{d}$ ) the solution is dilute; the single chain statistics in this regime is discussed in paper $I$ (see also Eq. (3.1)). At the overlap concentration $c_{*} \sim R^{-3}$, corresponding to

$$
\Phi_{*} \sim\left\{\begin{array}{ccc}
d^{2} / L^{2}, & \text { if } & L \lesssim l \\
d^{2} /\left(L l^{3}\right)^{1 / 2}, & \text { if } & l \lesssim L \lesssim L_{4} \\
\left(d^{7} l^{-3} L^{-4}\right)^{1 / 5}, & \text { if } & L \gtrsim L_{4}
\end{array}\right.
$$

the chains start to overlap (semidilute solution). The excluded volume part of the free energy (2.1) per chain in the isotropic phase in the mean field approximation is

$$
U_{\mathrm{int}}^{\mathrm{mf}} \sim \Phi L / d .
$$

Hence the chain statistics is strongly disturbed by the excluded volume interactions above the threshold

$$
\Phi_{* *} \sim d / L \text {. }
$$

The intermediate region $\left(\Phi_{*}<\Phi<\Phi_{* *}\right.$ ) corresponds to a nearly ideal solution, where the chains overlap geometrically, but do not interact (region $\Theta$ in Fig. 1). The correlation radius $\xi$ here coincides with the chain size:

$$
\xi \sim R \sim \min \left\{L,(L l)^{1 / 2}\right\} \quad \text { in region } \Theta(\text { for } \Omega \lesssim 1),
$$

where $R$ is determined by equation (3.1) (note that in the $\Theta$ region $L$ must be smaller than $L_{4}$ ). 
Above the threshold $\Phi_{* *}$ we find either a transition into a nematic phase if $L \lesssim l\left(\mathrm{~N}_{\mathrm{L}}\right.$ region), or a cross-over to the so called marginal solvent region if $l \lesssim L \lesssim L_{4}$ ( $\mathrm{I}_{\mathrm{m}}$ region). The $\Theta$-region does not exist when $L \gtrsim L_{4}$ as here one would have $\Phi_{* *}<\Phi_{*}$.

If $L \gtrsim L_{4}$ the chains interact strongly and are therefore swollen at the overlap threshold $\Phi=\Phi_{*}$. Therefore the region $L>L_{4}, \Phi>\Phi^{*}$ (region $I_{s}$ in Fig. 1) is the semidilute good solvent region. It is well-known that the properties of the solution in this region can be described in terms of concentration blobs [6]. The blob contour length $\Lambda$ is determined by the requirement that the blobs nearly tightly fill the volume of the system. Inside a blob the effect of the surrounding chains is negligible so that the spatial blob size, $\xi$, is determined by the single coil statistics: $\xi \sim\left(\Lambda^{3} d l\right)^{1 / 5}$ This leads to

$$
\Lambda \sim\left(\frac{d^{7}}{l^{3} \Phi^{5}}\right)^{1 / 4} \sim L_{4}\left(\frac{d^{3}}{l^{3} \Phi}\right)^{5 / 4}, \quad \xi \sim\left(\frac{d^{5}}{l \Phi^{3}}\right)^{1 / 4} \quad \text { for } \mathrm{I}_{\mathrm{s}} \text { region. }
$$

The typical free energy per blob is of order unity, and the radius of a chain in the $I_{s}$ region is

$$
R \sim \xi(L / \Lambda)^{1 / 2} \sim L^{1 / 2}\left(d^{3} l / \Phi\right)^{1 / 8}
$$

The excluded volume interactions are screened and the chains are Gaussian at scales larger than the correlation length $\xi$.

The swollen blob picture requires that $\Lambda \gtrsim L_{4}$, i.e.

$$
\Phi \lesssim \Phi_{\mathrm{m}} \equiv(d / l)^{3}
$$

Above $\Phi_{m}$ the solution is in the "marginal" regime, $I_{m}$, where the chain fragments are not swollen at any scales. Thus, the mean field approximation can be used in region $\mathrm{I}_{\mathrm{m}}$. Note that the chain size given by (3.7) crosses over to the ideal coil size $R \sim(L l)^{1 / 2}$ at the concentration $\Phi_{\mathrm{m}}$. The correlation length $\xi$ is then the "interaction blob" size. Using equation (2.6) we get

$$
\Lambda \sim d / \Phi ; \quad \xi \sim(l \Lambda)^{1 / 2} \sim(d l / \Phi)^{1 / 2} \text { and } R \sim(L l)^{1 / 2} \text { in region } \mathrm{I}_{\mathrm{m}}
$$

Note, that $\xi \sim R$ at the cross-over boundary between the $\Theta$ and $\mathrm{I}_{\mathrm{m}}$ regions, $\xi<R$ inside the $\mathrm{I}_{\mathrm{m}}$ region, and $\xi \sim l$ at the transition to the $\mathrm{N}$ phase (at $\Phi \sim d / l$, see Eq. (3.10) below).

3.2. Nematic Phase. - The isotropic-nematic transition, which occurs at higher concentrations, can be analyzed using equation (2.3). We recover below the well-known results of references $[14,16]$ from this approach.

The solid thick line in Figure 1 is the first order transition line from the isotropic to the nematic phase. If the chains are long enough $(L>l)$, the transition point is determined by the persistence length $l$; shorter fragments of persistent chains $(L \lesssim l)$ behave like rigid rods:

$$
\Phi_{\mathrm{IN}} \sim\left\{\begin{array}{lll}
d / L, & \text { if } & L \lesssim l \\
d / l, & \text { if } & L \gtrsim l
\end{array}\right.
$$

In both cases the transition is first order: the concentration difference between the coexisting isotropic and nematic phases is of the order of the transition concentration (the precise numerical prefactors cannot be calculated from the scaling approach):

$$
\Phi_{\mathrm{I}} \sim \Phi_{\mathrm{N}}-\Phi_{\mathrm{I}} \sim \Phi_{\mathrm{IN}} .
$$


Depending on the concentration and the length of the chains, the characteristic orientation angle $\gamma$ behaves either as in rod-like nematic solutions (the region $\mathrm{N}_{\mathrm{L}}$ ), or as in long semiflexible chain nematics (the region $\mathrm{N}_{\infty}$ ),

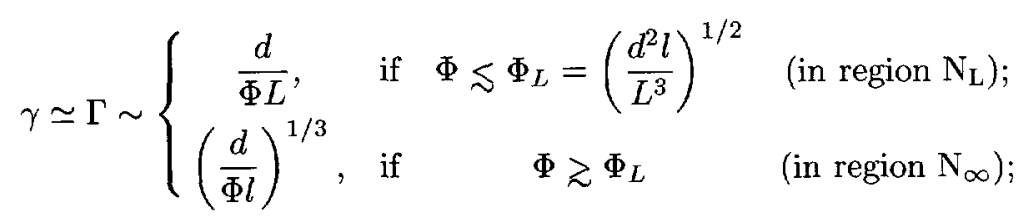

(these two formulae correspond to the cases when either the second term $\left(\ln \frac{1}{\Gamma}\right)$ or the third term $(L / l) \Gamma^{-2}$ correspondingly dominates in the sum (2.3)). The boundary between the regions $\mathrm{N}_{\mathrm{L}}$ and $\mathrm{N}_{\infty}$ is a cross-over line.

In the region $\mathrm{N}_{\mathrm{L}}$ the chains behave like rigid rods, hence the correlation lengths $\xi$ and the chain sizes $R$ are $\left({ }^{1}\right)$ :

$$
\xi_{z} \sim R_{z} \sim L ; \quad \xi_{x}=\xi_{y} \sim R_{x}=R_{y} \sim L \gamma \sim d / \Phi \quad \text { (in region } \mathrm{N}_{\mathrm{L}} \text { ) }
$$

Here the director (the axis of preferential orientation of the $\mathbf{t}$-vectors) is assumed to be parallel to $z$-axis (as in Eq. (I.4.2) and Fig. I.5b).

In the region $\mathrm{N}_{\infty}$, the correlation lengths and the chain sizes are ( $c f$. Ref. [17]):

$$
\begin{aligned}
& R_{x}=R_{y} \sim \gamma^{2}(L l)^{1 / 2} ; \xi_{x}=\xi_{y} \sim \gamma^{3} l \\
& R_{z} \sim \min \left\{L,\left(L l^{*}\right)^{1 / 2}\right\}
\end{aligned}
$$

where $\gamma \simeq(d / \Phi l)^{1 / 3}$ is given by equation (3.12),

$$
l^{*} \sim l \exp \left(\operatorname{const} \gamma^{-3 / 2}\right)
$$

is the effective persistence length in $z$-direction, and const, is a numerical constant $\left({ }^{2}\right)$. Equations $(3.14,3.15)$ are obtained using the following ideas: $(1)$ the transverse $(x, y)$ correlation length is equal to the corresponding size of the interaction blob; (2) the contour length of the interaction blob is $\Lambda \sim \frac{d}{\Phi \Gamma}$ (see Eq. (2.6)); (3) the chain statistics in the $x-y$ plane is Gaussian on scales larger than the interaction blob. For persistent chains the latter statement is not applicable to the chain size in the $z$-direction because of the induced nematic rigidity effect $\left(l^{*} \gg l\right)[17]\left({ }^{3}\right)$. The simplest scaling interpretation of equations $(3.15,3.16)$ is given in the Appendix.

Thus, we considered all the regions in the diagram of Figure 1. In the next sections we study the changes in this diagram which are due to the second rigidity $l_{2}$.

$\left({ }^{1}\right)$ The correlation lengths are related to the static structure factor, $S(\mathbf{q})=\left\langle\delta \phi_{\mathbf{q}} \delta \phi_{-\mathbf{q}}\right\rangle$, where $\delta \phi_{\mathbf{q}}=\int\left[\phi(\mathbf{r})-\phi_{0}\right] \mathrm{e}^{-\imath \mathbf{q} \mathbf{r}} \mathrm{d}^{3} r$, and $\phi(\mathbf{r})$ is the local monomer concentration; within the scaling accuracy the structure factor can be represented as $S(\mathbf{q})=S(0) /\left(1+q_{x}^{2} \xi_{x}^{2}+q_{y}^{2} \xi_{y}^{2}+q_{z}^{2} \xi_{z}^{2}\right)$.

$\left({ }^{2}\right)$ The correlation length $\xi_{z}$ is not always directly related to $l^{*}$ and $L$; we do not consider this length here.

$\left({ }^{3}\right)$ This effect is related to the fact that in a highly ordered nematic the regions of preferential orientations of $\mathbf{t}$ vectors (near $+z$ and near $-z$ ) are not topologically connected. 


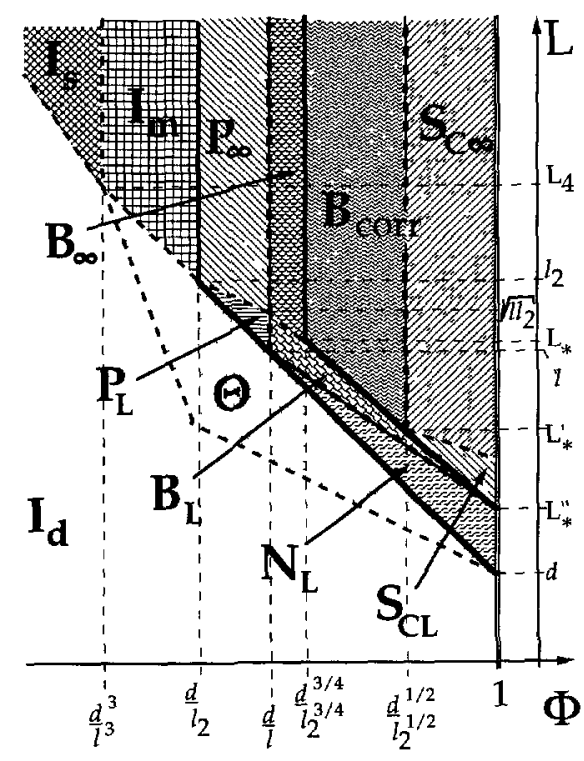

Fig. 2. - The same diagram as Figure 1 for the case of a low planar rigidity: $l<l_{2}<l(l / d)^{4 / 3}=l_{2}^{*}$. Thick dashed lines combined with thin solid lines correspond to second order transitions. Pancake nematics are denoted as $\mathrm{P}$; biaxial nematics as $\mathrm{B}$; smectics $\mathrm{C}$ as $\mathrm{S}_{\mathrm{C}} . L_{*}=l_{2}(d / l)^{2 / 3} ; L_{*}^{\prime} \sim l_{2}^{1 / 3} d^{2 / 3}$; $L_{*}^{\prime \prime} \sim l^{1 / 3} d^{2 / 3}$. Other notations as in the previous Figure 1. The particular case illustrated in this Figure is $l(l / d)^{2 / 3}<l_{2}<l_{2}^{*}$. The phase diagram for a lower second rigidity, $l<l_{2}<l(l / d)^{2 / 3}$ is similar: the only difference is that $L_{*}<l$ in the latter regime.

\section{Phase Diagram of Polymers with a Low Planar Rigidity:}

$$
l \lesssim l_{2} \lesssim l(l / d)^{4 / 3} \quad\left(d^{2} / l^{2} \lesssim \Omega \lesssim(d / l)^{2 / 3}\right)
$$

In this range of rigidity the various regimes for the isolated chain statistics are shown in Figure I.3b (or Fig. II.2a):

$$
\begin{array}{ccccc}
R_{\|} \sim L & \text { and } & R_{\perp} \sim d & \text { if } & d \lesssim L \lesssim L_{3}=\left(l_{2} d^{2}\right)^{1 / 3} \\
R_{\|} \sim L & \text { and } & R_{\perp} \sim L^{3 / 2} / l_{2}^{1 / 2} & \text { if } & L_{3} \lesssim L \lesssim l \\
R_{\|} \sim(L l)^{1 / 2} & \text { and } & R_{\perp} \sim L\left(l / l_{2}\right)^{1 / 2} & \text { if } & l \lesssim L \lesssim l_{2} \\
R_{\|} \sim(L l)^{1 / 2} & \text { and } & R_{\perp} \sim R_{\|} & \text {if } & l_{2} \lesssim L \lesssim L_{4} \\
R_{\|} \sim\left(L^{3} l d\right)^{1 / 5} & \text { and } & R_{\perp} \simeq R_{\|} & \text {if } & L_{4} \lesssim L .
\end{array}
$$

The shape of polymer coils is anisotropic only if the chain length is shorter than $l_{2}$; at larger scales the chains behave as ordinary persistent macromolecules. The diagram of states is shown in Figure 2. Below we discuss its specific features in comparison with the diagram of ordinary semiflexible chains (Fig. 1).

It is clear that the second rigidity is not important if the interaction blob contour length $\Lambda \gtrsim l_{2} ;$ in the isotropic phase, $\Lambda=d / \Phi$ and the second rigidity plays no role if $\Phi \lesssim d / l_{2}$. All the regimes in the region $\Phi \lesssim d / l_{2}$ are denoted in the same way in Figures 1 and 2 and their properties are described in Section 3 (Eqs. (3.2, 3.4-3.9)).

4.1. Pancake Nematic Phases. - Above the concentration $\Phi=d / l_{2}$, the chain anisotropy connected with the second rigidity becomes important. In this regime the chains are 
transparent (the solution is marginal), so that the mean field approximation is applicable. The ordering transitions can be studied using the free energy functional (2.2) where the correlation correction are neglected (below we verify that $\mathcal{N} \ll 1$ in the pancake nematic region). The minimization of the free energy (Eq. (2.2)), over $\Psi_{1}, \Psi_{2}$ and $\Gamma$ at a given $\Phi$ leads to the following results. If $L>l$ then as concentration is increased the isotropic $\left(\mathrm{I}_{\mathrm{m}}\right)$ phase transforms to a pancake nematic $(\mathrm{P})$ phase where there is no anisotropy connected with $\gamma(\Gamma=1)$, but where the PMF's are ordered: $\Psi_{1}=\Psi_{2}=\Psi<1$. The $I_{m} \rightarrow P$ transition takes place at

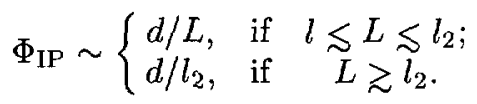

It is a first order transition with

$$
\Phi_{\mathrm{I}} \sim \Phi_{\mathrm{P}}-\Phi_{\mathrm{I}} \sim \Phi_{\mathrm{IP}}
$$

The transition is indicated in Figure 2 by a thick solid line.

The subregion $\mathrm{P}_{\mathrm{L}}$ of the pancake nematic region in the phase diagram, Figure 2, corresponds to shorter chains: the index $L$ here and below means that the properties of the system essentially depend on the chain length (see Eq. (3.12)). The crossover between the regions $\mathrm{P}_{\infty}$ and $P_{L}$,

$$
\Phi_{L} \sim d l_{2} L^{-2}
$$

is shown by a dashed line.

The order parameter inside the P-phase is:

$$
\Psi \sim \begin{cases}\left(\frac{d}{\Phi L}\right)^{1 / 2}, & \text { in region } \mathrm{P}_{\mathrm{L}} \\ \left(\frac{d}{\Phi l_{2}}\right)^{1 / 4}, & \text { in region } \mathrm{P}_{\infty}\end{cases}
$$

The results (4.2-4.5) are valid provided that the correlation correction (which is proportional to $\mathcal{N}$ ) is negligible, i.e. if $\mathcal{N}<\Psi^{2}$ (see Eq. (2.1)). Using equations $(4.5,2.4,2.6)$ it is possible to show that this condition is always satisfied if $l_{2}<l_{2}^{*}=l(l / d)^{4 / 3}$ (note that the worst situation corresponds to the upper right corner of the $\mathrm{P}_{\mathrm{L}}$ region). Therefore the correlation correction is indeed negligible in the P-nematic phase when $\left(d^{2} / l^{2} \lesssim \Omega \lesssim(d / l)^{2 / 3}\right)$.

The correlation lengths in the pancake nematic phase are of order of the sizes of an interaction blob with contour length $\Lambda \sim d / \Phi$ (see Eq. (2.6)): $\xi_{z}=R_{\|}(\Lambda), \xi_{x} \sim \Psi \xi_{z}$, where $\Psi$ is given by equation (4.5). Taking into account that the statistics in the PMF $(y-z)$ plane is Gaussian (see Eqs. (4.1), note that $d / l_{2}<\Phi<d / l$, hence $l<\Lambda<l_{2}$ ) we get

$$
\xi_{y}=\xi_{z} \sim(l \Lambda)^{1 / 2} \sim(l d / \Phi)^{1 / 2} ; \quad \xi_{x} \sim \begin{cases}\frac{d}{\Phi}\left(\frac{l}{L}\right)^{1 / 2}, & \mathrm{P}_{L} \\ \left(\frac{l^{2} d^{3}}{l_{2} \Phi^{3}}\right)^{1 / 4}, & \mathrm{P}_{\infty}\end{cases}
$$

On scales larger than $\Lambda$ the chain statistics is Gaussian, so that $R_{z} \sim \xi_{z}\left(\frac{L}{\Lambda}\right)^{\frac{1}{2}}, R_{x} \sim \xi_{x}\left(\frac{L}{\Lambda}\right)^{\frac{1}{2}}$, i.e.

$$
R_{y}=R_{z} \sim(L l)^{1 / 2} ; \quad R_{x} \sim\left\{\begin{array}{cc}
\left(\frac{d l}{\Phi}\right)^{1 / 2} & , \mathrm{P}_{\mathrm{L}} \\
(L l)^{1 / 2}\left(\frac{d}{\Phi l_{2}}\right)^{1 / 4} & , \mathrm{P}_{\infty}
\end{array}\right.
$$

The chain conformation is always anisotropic in the $\mathrm{P}_{\infty}$ and $\mathrm{P}_{\mathrm{L}}$ nematic regions: $R_{x}<R_{z}$. 
4.2. Biaxial Nematic Phases. - At even higher concentrations, $\Phi>d / l$, an additional orientation of the t-vectors "inside" the PMF's (which are nearly parallel to $y-z$ plane) becomes favorable, so that the stable phase is a biaxial nematic phase $\left(\Gamma<1, \Psi_{1}<1, \Psi_{2}<1\right)$. The characteristics of the biaxial (B) phase are found by minimization of equation (2.2). In particular we locate the $\mathrm{P} \rightarrow \mathrm{B}$ transition at

$$
\Phi_{\mathrm{PB}} \sim d / l
$$

and the orientation angle $\gamma$ in the region $\Phi>\Phi_{\mathrm{PB}}$ is

$$
\gamma \sim \Gamma \simeq\left(\frac{d}{\Phi l}\right)^{1 / 3}
$$

Here we supposed that the correlation corrections are small in this region $(\mathcal{N} \ll 1)$; this statement is checked below. Note that within the scaling accuracy adopted in this paper it is not possible to discriminate between a 1st order and a 2nd order phase transitions. An additional analysis based on the second virial approximation only shows that the $\mathrm{P} \rightarrow \mathrm{B}$ transition is second order $\left({ }^{4}\right)$.

Neglecting the correlation corrections we also get other orientation parameters:

$$
\begin{array}{ll}
\Psi_{1} \sim\left(\frac{d^{2} l}{\Phi^{2} L^{3}}\right)^{1 / 6} ; & \Psi_{2} \sim\left(\frac{d^{4}}{\Phi^{4} L^{3} l}\right)^{1 / 6} \quad \text { in region } \mathrm{B}_{\mathrm{L}} \\
\Psi_{1} \sim\left\{\begin{array}{ll}
\left(\frac{d^{2} l}{\Phi^{2} L^{3}}\right)^{1 / 6}, & \Phi<\Phi^{\prime} \\
\left(\frac{d^{2} l}{\Phi^{2} l_{2}^{3}}\right)^{1 / 12}, & \Phi>\Phi^{\prime}
\end{array} \Psi_{2} \sim\left(\frac{d^{4}}{\Phi^{4} l_{2}^{3} l}\right)^{1 / 12} \text { in region } \mathrm{B}_{\infty}\right.
\end{array}
$$

where $\Phi^{\prime} \sim \frac{d}{L^{3}}\left(l l_{2}^{3}\right)^{1 / 2}$ Note that $\Psi_{1} \gtrsim \Psi_{2}$ for $\Phi \gtrsim d / l$ in all the above regimes. The crossover between the regions $B_{L}$ and $B_{\infty}$,

$$
\Phi_{L} \sim\left(\frac{d^{4} l_{2}^{3}}{l L^{6}}\right)^{1 / 4}
$$

is shown in Figure 2 by dashed line.

Let us now calculate the correlation corrections in the B phase. First we note that the interaction blob length $\Lambda \sim \frac{d}{\Phi \gamma} \sim l^{1 / 3}\left(\frac{d}{\Phi}\right)^{2 / 3}$ is smaller than the persistence length $l$ since $\Phi>d / l$. Therefore $\mathcal{N}$ is the number of secondary contacts between nearly straight fragments of length $\Lambda \ll l$. The typical angle between these fragments at the priming intersection point is $\gamma$. In order to intersect again at least one of the fragments must bend on an angle of order $\gamma$. The bending energy is $U \sim l \gamma^{2} / \lambda$, where $\lambda$ is the contour distance between the primary and the secondary contact. With the obvious condition $U \lesssim 1$ we thus get $\lambda \gtrsim l \gamma^{2}$. Noting that $l \gamma^{2} \sim \Lambda$ we conclude that $(i)$ the number of secondary contacts, $\mathcal{N}$, cannot be large; (ii) the dominant contribution to $\mathcal{N}$ comes from scales $\lambda \sim \Lambda$. With a high second rigidity $l_{2} \gg l \gg \Lambda$, the conformation of a $\Lambda$-fragment is flat: it is restricted to a plain angle $\sim \gamma$. A secondary contact between two $\Lambda$-fragments is possible only if the corresponding plain angles do intersect. This geometric condition means that $\psi_{2} \lesssim \gamma \psi_{1}$. An analysis of the general free energy expression (Eq. (2.2)) shows that it is never favorable to decrease $\psi_{2}$ well below $\gamma \psi_{1}$.

$\left({ }^{4}\right)$ This transition is actually quite similar to the nematic ordering of ordinary persistent macromolecules under an external orientational field of the "easy plane" type [19]. 
Therefore the last condition can be rewritten as $\psi_{2} \sim \gamma \psi_{1}$. With this condition the probability of a secondary contact can be easily estimated as the ratio of the chain thickness $d$ to the typical projection of a $\Lambda$-fragment onto the $x$-axis, $R_{x}(\Lambda) \sim \psi_{1} \Lambda \gamma$ :

$$
\mathcal{N} \sim \frac{d}{\psi_{1} \Lambda \gamma} \sim \frac{\Phi}{\psi_{1}} \sim \frac{\Phi \gamma}{\psi_{2}}
$$

The correlation correction to the interaction energy $-\frac{L}{d} \Phi \Gamma \mathcal{N}$, should be compared with the dominant angular dependent terms, $\frac{L}{d} \Phi \Gamma\left(\Psi_{1}^{2}+\Psi_{2}^{2} / \Gamma^{2}\right.$ ) (in Eq. (2.2)). Thus the correlation correction is important if $\mathcal{N}>\psi_{1}^{2}+\psi_{2}^{2} / \Gamma^{2}\left(^{5}\right)$. Using equations $(4.10,4.12)$ with the latter condition we get $\Phi>\Phi_{\text {corr }}$, where

$$
\Phi_{\text {corr }} \sim\left(\frac{l}{l_{2}}\right)^{3 / 4}
$$

In the region $\Phi>\Phi_{\text {corr }}$ (which is denoted as $B_{\text {corr }}$ in Fig. 2) the correlation correction dominates the angular dependence of the interaction energy. In the $\mathrm{B}_{\mathrm{cor}}$ region, the orientation angles, obtained by minimization of equation (2.2), are

$$
\gamma \sim\left(\frac{d}{\Phi l}\right)^{1 / 3}, \quad \psi_{1} \sim \frac{l}{l_{2} \Phi}, \quad \psi_{2} \sim \gamma \psi_{1} \sim\left(\frac{d l^{2}}{l_{2}^{3} \Phi^{4}}\right)^{1 / 3} \quad \text { in } B_{\text {corr }} \text { phase }
$$

With the free energy expression (Eq. (2.2)) it is possible to show that the regions $\mathrm{B}_{\infty}$ and $\mathrm{B}_{\text {corr }}$ are separated by a weak first order transition line at $\Phi \sim \Phi_{\text {corr }}$. The phase separation region, $\Delta \Phi$, is relatively narrow:

$$
\Delta \Phi / \Phi_{\text {corr }} \sim\left(\frac{l}{l_{2}}\right)^{1 / 2}
$$

Equations $(4.13,4.15)$ are valid if $\Phi_{\text {corr }}>\Phi_{L}$, i.e. if $L>L_{*}$, where

$$
L_{*} \sim l_{2}\left(\frac{d}{l}\right)^{2 / 3}
$$

If $L<L^{*}$ then the $\mathrm{B}_{\text {corr }}$ phase appears before the $\mathrm{B}_{\mathrm{L}} \rightarrow \mathrm{B}_{\infty}$ transition. The corresponding $\mathrm{B}_{\mathrm{L}} \rightarrow \mathrm{B}_{\mathrm{corr}}$ transition is similar to the one considered before; the transition concentration and the relative width of the transition region are

$$
\Phi_{\text {corr }} \sim\left(\frac{l}{l_{2}}\right)^{3 / 4}\left(\frac{L_{*}}{L} \ln \frac{L_{*}}{L}\right)^{3 / 8} ; \frac{\Delta \Phi}{\Phi_{\text {corr }}} \sim\left(\frac{l}{l_{2}}\right)^{1 / 2}\left(\frac{L_{*}}{L} \ln \frac{L_{*}}{L}\right)^{3 / 4}
$$

The correlation lengths and the coil sizes for a biaxial nematic can be found in exactly the same way as for the uniaxial nematic (see Sects. 3.2, 4.1). The interaction blob length is $\Lambda \sim \frac{d}{\Phi \gamma} \sim l \gamma^{2}$, and $\xi_{y} \sim \gamma \Lambda ; R_{y} \sim \xi_{y}\left(\frac{L}{\Lambda}\right)^{1 / 2}$ The chain size in the direction of ordering of the t vectors ( $z$-direction) is determined by the induced chain rigidity, as in a usual semiflexible polymer nematic phase (see Eq. (3.16)). The size in the $x$-direction, $R_{x}$, is determined by the angle $\psi_{2}$ between the PMF and $z$-axis, and the "persistence length" $l_{x}^{*}$ in the $x$-direction which corresponds to the $\left\langle t_{x}(0) t_{x}(s)\right\rangle$ correlation function. These orientation correlations decay

$\left({ }^{5}\right)$ Note that the r.h.s. of this inequality is small, so that the correlation correction might be important even if $\mathcal{N} \ll 1$. 
either because of chain bending in the $y-z$ plane $\left(l^{*}\right)$ or by bending in the $x-z$ plane (the corresponding scale is $l_{2} \psi_{2}^{2}$ - on this scale the chain bends on an angle of order $\psi_{2}$ ). Therefore

$$
l_{x}^{*}=\min \left\{l^{*}, l_{2} \psi_{2}^{2}\right\}
$$

where $l^{*}$ is determined by equation (3.16). Thus we get

$$
\begin{gathered}
\xi_{y} \sim \frac{d}{\Phi} ; \quad R_{y} \sim \gamma^{2}(l L)^{1 / 2} \\
R_{z} \sim \min \left\{L,\left(L l^{*}\right)^{1 / 2}\right\} ; \quad R_{x} \sim \psi_{2} \min \left\{L,\left(L l_{x}^{*}\right)^{1 / 2}\right\} \quad \text { in B phases. }
\end{gathered}
$$

Note that well inside the B region $\left(\Phi \gg \Phi_{\mathrm{PB}} \sim d / l\right)$ the orientation angle $\gamma$ is small, so that $l^{*}$ is exponentially large. In this case $R_{z} \sim L$, and $l_{x}^{*}=l_{2} \psi_{2}^{2}$, so that $l_{x}^{*}>L$ in the $\mathrm{B}_{\mathrm{L}}$ and $l_{x}^{*}<L$ in the $\mathrm{B}_{\infty}$ or $\mathrm{B}_{\text {corr }}$ regions. Therefore

$$
R_{x}=\left\{\begin{array}{ccc}
\psi_{2} L & \mathrm{~B}_{\mathrm{L}} \\
\psi_{2}^{2}\left(L l_{2}\right)^{1 / 2} & , & \mathrm{~B}_{\infty}+\mathrm{B}_{\text {corr }}
\end{array}\right.
$$

4.3. Nematic Phase. - If $L<l$, the polymer chains are rod-like. In this case as the concentration is increased up to $\Phi_{\text {IN }} \sim d / L$, the solution orders in the classical nematic phase considered in Section 3. The properties of the $\mathrm{N}_{\mathrm{L}}$ phase which appear as a result of this transition are independent of both persistence lengths $l$ and $l_{2}$. The orientation angle $\gamma \sim \frac{d}{\Phi L}$ (see Eq. (3.12)) is decreasing as concentration is further increased. At $\Phi \sim \Phi_{L}=\left(\frac{d^{2} l}{L^{3}}\right)^{1 / 2}$ the orientation angle becomes of order of the typical bending angle $\gamma_{0} \sim\left(\frac{L}{l}\right)^{1 / 2}$, so that the flexibility of polymer chains becomes important for $\Phi>\Phi_{L}$. In this region the typical interaction energy per chain $\frac{L}{d} \Phi \gamma$ (see Eq. (2.2)) becomes large, and the minimum of the free energy, Eq. (2.2), corresponds to the biaxial nematic phase $\mathrm{B}_{\mathrm{L}}$ with orientation angles given by equations (4.8), (4.9). A phase transition $\mathrm{N}_{\mathrm{L}} \rightarrow \mathrm{B}_{\mathrm{L}}$ occurs when

$$
\Phi_{\mathrm{NB}}=\left(\frac{d^{2} l}{L^{3}}\right)^{1 / 2}, \quad L<l .
$$

We expect that this is a second order phase transition (as the symmetry of the additional order parameter related to biaxiality is compatible with a second order phase transition).

4.4. Smectic Phase. - Let us consider the $\mathbf{B}_{\text {corr }}$ phase. The number of secondary contacts in this phase is (see Eqs. $(4.12,4.14)): \mathcal{N} \sim \frac{l_{2}}{l} \Phi^{2}$; therefore $\mathcal{N} \sim 1$ for $\Phi \sim \Phi_{\mathrm{BS}} \sim\left(\frac{l}{l_{2}}\right)^{1 / 2}$ Simultaneously at $\Phi \sim \Phi_{\mathrm{BS}}$ the $x$-projection of the interaction blob $R_{x}(\Lambda)$ becomes of the order of the chain thickness $d$. In the region $\Phi>\Phi_{\mathrm{BS}}$ the condition $R_{x}(\lambda) \sim d$ defines the chain fragment length $\lambda$ which is larger than $\Lambda$. In this region an arrangement of the chains in parallel layers of thickness $\sim d$ is favorable: as shown in paper $\mathrm{I}$, a layered smectic structure is characterized by a lower interaction energy. The interaction energy gain, $\Delta F_{\text {int }}$, is of order 1 per interaction blob. On the other hand the increase of the free energy, $\Delta F_{\text {ord }}$, related to entropy loss due to smectic ordering is of order 1 per fragment $\lambda$. Therefore $\Delta F_{\text {int }} \propto \frac{1}{\Lambda}>\Delta F_{\text {ord }} \propto \frac{1}{\lambda}$. Since the $\mathbf{t}$ vectors of the chains also order in each layer, the corresponding structure is a smectic $C\left(\mathrm{~S}_{\mathrm{C}}\right)$ phase. Thus we predict $\mathrm{B}_{\text {corr }} \rightarrow \mathrm{S}_{\mathrm{C}}$ transition at

$$
\Phi_{\mathrm{BS}} \sim\left(\frac{l}{l_{2}}\right)^{1 / 2}
$$


which is presumably a second-order transition (according to the symmetry change associated with the transition).

So far we have assumed that the chain length $L$ is larger than the interaction blob $\Lambda \sim \frac{d}{\Phi \gamma}$ : $L>\Lambda$. Using equations (4.22) and (4.8) we rewrite this condition as $L>L_{*}^{\prime}=l_{2}^{1 / 3} d^{2 / 3}$. For shorter chains, $L<L_{*}^{\prime}$, the concentration of the nematic-smectic transition is defined by a balance between the interaction energy gain $\Delta \mathcal{F}_{\text {int }} \sim \frac{L}{\Lambda}$ (per chain), and the loss of orientational entropy which is dominated by the "ideal gas" term (second term in the r.h.s. of Eq. (2.2)), $\Delta \mathcal{F}_{\text {ord }} \sim \ln \frac{\psi_{1}(\mathrm{~B}) \psi_{2}(\mathrm{~B})}{\psi_{1}(\mathrm{~S}) \psi_{2} \text { (S) }}$, where $\psi_{1}$ (B) is the orientation angle in the $\mathrm{B}$ phase at the transition point, etc., and we take into account the fact that the angle $\gamma$ is not affected much by the transition. The condition $\Delta \mathcal{F}_{\text {int }} \sim \Delta \mathcal{F}_{\text {ord }}$ thus defines the transition concentration

$$
\Phi_{\mathrm{BS}} \sim\left(\frac{d^{2} l}{L^{3}}\right)^{1 / 2}\left(\ln \frac{L^{3}}{d^{2} l}\right)^{3 / 2},\left(d^{2} l\right)^{1 / 3} \equiv L_{*}^{\prime \prime}<L<L_{*}^{\prime} \equiv\left(d^{2} l_{2}\right)^{1 / 3}
$$

Equation (4.23) defines the line of the first order phase transition $B_{L} \rightarrow S_{C}$. The transition is accompanied by a jump of $\psi_{1}$ from $\sim 1$ to $\psi_{1} \sim \Phi \sim\left(\frac{d^{2} l}{L^{3}}\right)^{1 / 2}$, and $\psi_{2}$ from $\sim \gamma$ to $\psi_{2} \sim \gamma \Phi$ (here we omit logarithmic factors). The relative width of the corresponding phase separation region is $\Delta \Phi / \Phi_{\mathrm{BS}} \sim 1$. The properties of the smectic phase are considered in more detail below in Section 6.4.

\section{Phase Diagram for the Case of Intermediate Planar Rigidity: $l(l / d)^{4 / 3} \lesssim l_{2} \lesssim l^{3} / d^{2} \quad\left((d / l)^{2 / 3} \lesssim \Omega \lesssim 1\right)$}

In this range of rigidity, the conformation of an isolated chain is still described by equation (4.1), Figure I.3b. The phase diagram for this regime, shown in Figure 3, is similar to the previous phase digram (see Fig. 2). The only difference is related to the fact that the correlation corrections are increasing as $l_{2}$ is increased. They become important at lower concentrations, in the $\mathrm{P}$-nematic region. Thus a new phase $P_{\text {corr }}$ appears on the diagram and the $\mathrm{B}_{\infty}$ region disappears: the correlations are always important in the $\mathrm{B}$ state if $L$ is large enough.

The transitions between $P_{\text {corr }}$ and the other pancake nematic phases can be studied using equations $(2.1,2.4,2.6)$; the factor $\frac{1}{1+\mathcal{N}}$ in the last interaction term in the r.h.s. of equation (2.1) can be approximated as $1-\mathcal{N}$ since $\mathcal{N}$ is always small in all $P$ regions (see Eq. (5.2)). The orientation angle $\psi$ in the $P_{\text {corr }}$ state is determined by the competition between the entropic term $\frac{L}{l_{2}} \Psi^{-2}$ and the correlation correction (Eq. (2.4)):

$$
\psi \sim \frac{l^{3 / 2}}{l_{2} \Phi^{1 / 2} d^{1 / 2}}, \quad \text { in } \mathrm{P}_{\text {corr }} .
$$

The orientation angle in the $P_{L}$ and $P_{\infty}$ states is defined in equation (4.5). Using equations $(5.1,2.4,2.6)$ we get the number of secondary contacts

$$
\mathcal{N} \sim \Omega=l_{2} d^{2} / l^{3}
$$

in the pancake nematic $P_{\text {corr }}$. The boundary between the regions $\mathrm{P}_{\infty}$ and $\mathrm{P}_{\text {corr }}$ is a crossover (shown as a dashed line in Fig. 3) at

$$
\Phi_{\text {corr }} \sim \frac{l^{6}}{d^{3} l_{2}^{3}}, L>L_{*}
$$




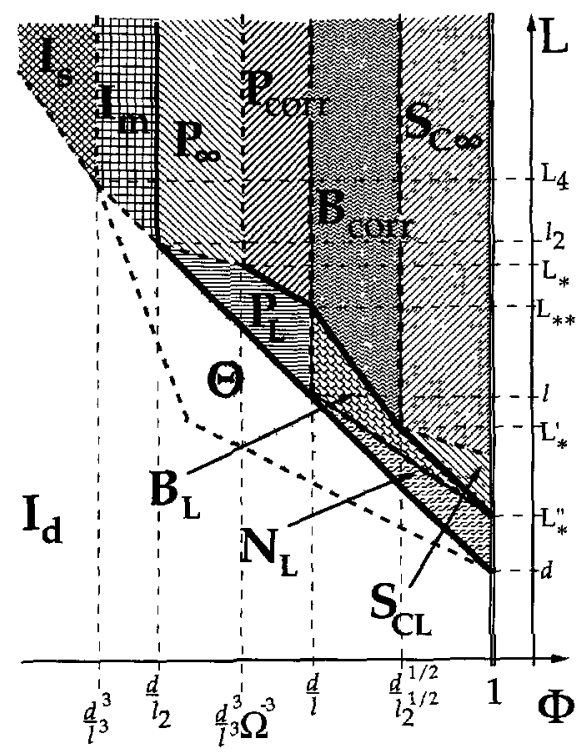

Fig. 3. - The same diagram as Figure 2 for the case of an intermediate planar rigidity: $l_{2}^{*}<l_{2}$ $<l^{3} / d^{2} . L_{*}=l_{2}^{2} d^{2} / l^{3} ; L_{* *}=\frac{l^{4}}{l_{2} d^{2}} \ln \frac{l_{2}}{l_{2}^{*}}$. Other notations as in the previous Figures $1-2$.

where

$$
L_{*}=l_{2} \Omega=l_{2}^{2} d^{2} / l^{3}
$$

The boundary between the $\mathrm{P}_{\mathrm{L}}$ and $\mathrm{P}_{\text {corr }}$ regions corresponds to a weak first-order transition at

$$
\Phi_{\text {corr }} \sim \frac{l^{3}}{L l_{2} d} \ln \frac{l_{2} \Omega}{L}, \quad L<L_{*}
$$

The relative width of the phase separation region is small

$$
\Delta \Phi / \Phi_{\text {corr }} \sim \Omega
$$

The phase transition line ends at the critical point $\Phi_{\mathrm{c}} \sim l^{6} /\left(d^{3} l_{2}^{3}\right), L_{\mathrm{c}} \sim L_{*}$. The regions $\mathrm{P}_{\mathrm{L}}$ and $\mathrm{P}_{\infty}$ are separated by a crossover line which is determined by equation (4.4).

The $\mathrm{P}_{\text {corr }}$ phase is characterized by the same chain sizes and correlation lengths in the $y-z$ plane as the other pancake nematic phases (see Eqs. $(4.6,4.7)$ ). The correlation length along the director is $\xi_{x} \sim \psi \xi_{z}$, and the corresponding size is $R_{x} \sim \xi_{x}\left(\frac{L}{\Lambda}\right)^{1 / 2}$

$$
\xi_{x} \sim \frac{l^{2}}{l_{2} \Phi} ; \quad R_{x} \sim \frac{l^{2}}{l_{2}}\left(\frac{L}{\Phi d}\right)^{1 / 2} \quad \text { in } \mathrm{P}_{\text {corr }} \text { phase. }
$$

The characteristics of all the other phases (I, B, N and S) are the same as in the regime of lower rigidity, $l_{2}<l(l / d)^{4 / 3}$ 


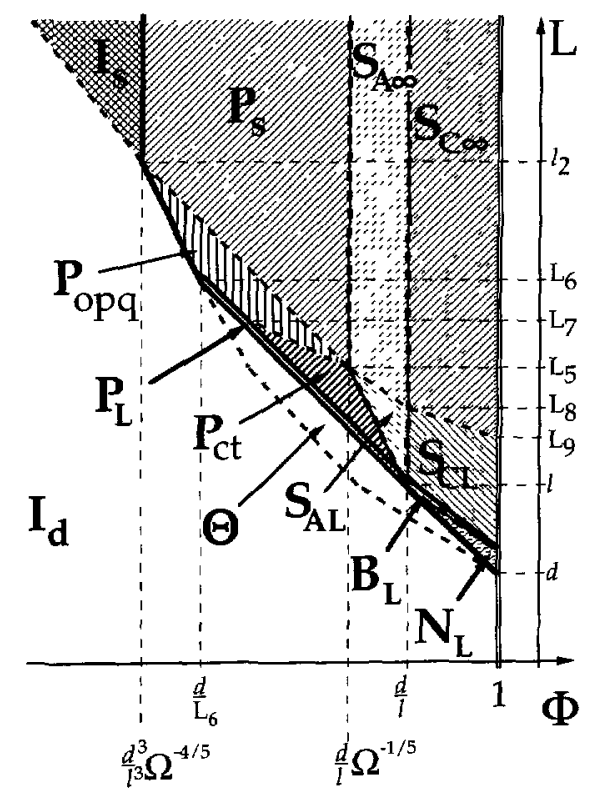

Fig. 4. - The same diagram as Figure 3 for the case of a high planar rigidity: $l_{2}>l^{3} / d^{2}$. Smectics $A$ are denoted as $S_{A}$. Other notations as in the previous Figures 1-3.

\section{Phase Diagram for the Case of High Planar Rigidity: $l^{3} / d^{2} \lesssim l_{2} \quad(1 \lesssim \Omega)$}

The dimensions of an isolated coil in this regime are shown in Figure I.3c, see also equation (I.3.11) and Figure II.2b:

$$
\begin{array}{ccccc}
R_{\|} \sim L & \text { and } & R_{\perp} \sim d & \text { if } & d \lesssim L \lesssim l \\
R_{\|} \sim l(L / l)^{3 / 4} & \text { and } & R_{\perp} \sim d & \text { if } & l \lesssim L \lesssim L 5 \equiv l \Omega^{2 / 5} \\
R_{\| \sim(L l)^{1 / 2} \Omega^{1 / 10}} & \text { and } & R_{\perp} \sim(L d / l) \Omega^{-2 / 5} & \text { if } & L_{5} \lesssim L \lesssim l_{2} \\
R_{\|} \sim\left(L^{3} l d\right)^{1 / 5} & \text { and } & R_{\perp} \simeq R_{\|} & \text {if } & l_{2} \lesssim L .
\end{array}
$$

The scaling description of Section I.3.3 of paper I is valid in this case. Note that different parts of the same chain strongly repel each other, hence the "coins" form "monisto" of nearly parallel coins if $L<l_{2}$, and for $L>l_{2}$ the polymer can be considered as a hyperchain of "balls" with strong excluded volume interactions, each ball being a monisto (see Fig. I.4 and discussion in Sect. I.3.3). The diagram of regimes for $\Omega \gtrsim 1$ shown in Figure 4 is considered in detail below.

6.1. Isotropic Phase. - At very low concentrations the chains are isolated; their properties in dilute solutions are described in Section I.3.3 (dilute region $\mathbf{I}_{\mathbf{d}}$ ). The semidilute regimes lay above the characteristic concentration $c_{*} \sim R_{\|}^{-3}$, where the chain overlap geometrically:

$$
\Phi_{*} \sim\left\{\begin{array}{ccc}
d^{2} / L^{2} & \text { if } & L \lesssim l \\
d^{2} L^{-5 / 4} l^{-3 / 4} & \text { if } & l \lesssim L \lesssim L_{5} \\
d^{2}\left(L l^{3}\right)^{-1 / 2} \Omega^{-3 / 10} & \text { if } & L_{5} \lesssim L \lesssim l_{2} \\
\left(d^{7} l^{-3} L^{-4}\right)^{1 / 5} & \text { if } & L \gtrsim l_{2} .
\end{array}\right.
$$


When $L \gtrsim l_{2}$, the chains are swollen three-dimensional coils, so at $\Phi \gtrsim \Phi_{*}$, in the region $I_{s}$, the properties of the solution can be described via the standard blob picture of semidilute solutions given in Section 3 (Eqs. $(3.6,3.7)$ ). Equations $(3.6,3.7)$ are valid if the blobs are isotropic, i.e. if $\Lambda>l_{2}$. This condition imposes that

$$
\Phi<\Phi_{\mathrm{IP}} \sim\left(\frac{d}{l}\right)^{3} \Omega^{-4 / 5}
$$

(see (3.8)). For $\Phi \gtrsim \Phi_{\mathrm{IP}}$ the blob size is smaller than $l_{2}$, so that the blobs are anisotropic. This leads to a drastic change of the properties of the solution: we show below, that a first order transition to a P-nematic phase takes place at $\Phi_{\mathrm{IP}}$.

When $l \lesssim L \lesssim l_{2}$ the isolated coil shape is disc-like (for $L \lesssim l$ it is rod-like), so we can expect a transition to a P-nematic (or an N-nematic for $L \lesssim l$ ) phase above $\Phi_{*}$. The meanfield approximation is valid if the typical energy of interaction between two coils, $U$, is small: $U \lesssim 1$. As discussed in paper II (Sect. II.2 and Appendices A, B) this condition is fulfilled if $L \lesssim L_{6}$ (see Eq. (II.2.11)). Therefore in the regime $L \lesssim L_{6}$ the interaction energy per chain can be estimated as $U_{\text {int }} \sim \Phi L / d$ (see Eqs. $(3.3,2.1)$ ) and the excluded volume interactions are negligible below the threshold $\Phi_{* *}=d / L$ (Eq. (3.4)). Thus the region between the lines $\Phi_{*}$ (Eq. (6.2)) and $\Phi_{* *}, \Phi_{*} \lesssim \Phi \lesssim \Phi_{* *}\left(L \lesssim L_{6}\right)$ corresponds to the $\Theta$ regime, where the chains overlap geometrically but do not interact appreciably.

In the $\Theta$ region the correlation length coincides with the chain size $R_{\|}$, given by equations (6.1):

$$
\xi \sim R_{\|} \sim \min \left\{L,\left(l L^{3}\right)^{1 / 4},(l L)^{1 / 2} \Omega^{1 / 10}\right\} \quad \text { in } \Theta \text { region, } \Omega \gtrsim 1 .
$$

For $l \lesssim L \lesssim L_{6}$ the line $\Phi_{* *}$ (Eq. (3.4)) is the transition line to a P-phase: $\Phi_{\text {IP }} \sim \Phi_{* *} \sim d / L$. For $L \lesssim l$ the line $\Phi_{* *}$ is the $\mathrm{I} \rightarrow \mathrm{N}$ transition line. Both transitions $(\mathrm{I} \rightarrow \mathrm{P}$ and $\mathrm{I} \rightarrow \mathrm{N})$ can be studied in a standard way using the free energy expression (Eqs. $(2.1,2.3)$ ). These are rather strong first order transitions: the phase separation region is wide $\Delta \Phi / \Phi_{\mathrm{IP}} \sim 1, \Delta \Phi / \Phi_{\mathrm{IN}} \sim 1$ (cf. Eqs. $(4.3,3.11)$ ).

For $L_{6} \lesssim L \lesssim l_{2}$ the interaction between the chains is rather strong already at the overlap concentration $\Phi_{*}: \quad U \sim\left(L d^{2} / l^{3}\right)^{1 / 2} \Omega^{-3 / 10} \gtrsim 1$ for $\psi \sim 1$ (see e.g. Eq. (II.A4)). Hence the chains interact like solid discs of size $R_{\perp} R_{\|}^{2}$ and form a P-nematic at $\Phi_{\mathrm{IP}} \sim \Phi_{*} \sim d^{2}\left(L l^{3}\right)^{-1 / 2} \Omega^{-3 / 10}$ (see Eq. (6.2)); note that the corresponding concentration of chains $\left.c_{\mathrm{IP}} \sim 1 / R_{\|}^{3}\right)$. This is again a first order phase transition, with $\Delta \Phi / \Phi_{\mathrm{IP}} \sim 1$.

To summarize, the transition from the isotropic to a $\mathrm{P}$-nematic phase occurs at

$$
\Phi_{\mathrm{IP}} \sim\left\{\begin{array}{clcl}
d / L, & \text { if } & l \lesssim L \lesssim L_{6} ; & \mathrm{I} \rightarrow \mathrm{P}_{\mathrm{L}} \\
d^{2}\left(L l^{3}\right)^{-1 / 2} \Omega^{-3 / 10}, & \text { if } & L_{6} \lesssim L \lesssim l_{2} ; & \mathrm{I} \rightarrow \mathrm{P}_{\mathrm{opq}} \\
(d / l)^{3} \Omega^{-4 / 5}, & \text { if } & L \gtrsim l_{2} ; & \mathrm{I} \rightarrow \mathrm{P}_{\mathrm{s}}
\end{array}\right.
$$

\subsection{Pancake Nematic Phase}

6.2.1. The Region $\mathrm{P}_{\text {opq }}$ - - We start the discussion with the case where the polymer chains behave like opaque discs, i.e. when the characteristic energy between two overlapping chains is of the order or larger than unity, $U \gtrsim 1$ (the $\mathrm{P}_{\mathrm{opq}}$ region in the diagram of Fig. 4).

The orientation angle $\psi$ in the $\mathrm{P}_{\text {opq }}$ phase is determined by the geometrical condition $c R_{\|}^{3} \psi \sim 1$, where

$$
V_{\psi} \sim R_{\|}^{3} \psi
$$


is the effective volume per chain $\left({ }^{6}\right)$ :

$$
\psi \sim\left(c R_{\|}^{3}\right)^{-1} \sim\left(\frac{d^{4}}{L l^{3}}\right)^{1 / 2} \Omega^{-3 / 10} \Phi^{-1} \quad \text { in region } \mathbf{P}_{\mathrm{opq}}
$$

(we assume here that $L \gtrsim L_{5}$ ). Each disc "touches" approximately one neighboring disc; the interaction free energy per disk is of order unity.

Thus in this regime the chain coincides with both the concentration blob and the interaction blob: $\Lambda \sim L$. As a result, the characteristic correlation lengths coincide with the chain dimensions, which are practically undisturbed by the interactions with surrounding chains (in the $\mathrm{P}_{\text {opq }}$ phase the chains rotate as a whole, but their shape remains the same as for isolated coils (see Eq. (6.1)):

$$
\begin{gathered}
\xi_{y}=\xi_{z} \simeq R_{y}=R_{z} \equiv R_{\mid j} \sim(L l)^{1 / 2} \Omega^{1 / 10} \\
\xi_{x} \simeq R_{x} \simeq \psi R_{\|} \sim \frac{d^{2}}{l \Phi} \Omega^{-1 / 5} \quad \text { in region } \mathrm{P}_{\mathrm{opq}} .
\end{gathered}
$$

Note that the correlations are strongly asymmetric: $\xi_{x} / \xi_{y} \ll 1$ ( $\xi_{x}$ becomes of order $\xi_{y}$ only at the left boundary of the $\mathrm{P}_{\text {opq }}$ region, at the $\Phi_{\mathrm{IP}}$ transition line).

The above treatment is valid if the chains are both opaque $(U>1)$ and rigid $\left(\psi>\psi_{0} \sim\right.$ $\left(\frac{L}{l_{2}}\right)^{1 / 2}$ ). Using equation (II.A4) (see paper II) and equation (6.7) we find that the opacity condition is valid if

$$
\Phi>\frac{l d}{L^{2}} \Omega^{3 / 5} \equiv \Phi_{o c}
$$

The rigidity condition $\left(\psi \gtrsim \psi_{0}\right)$ is equivalent to $\Phi \lesssim \Phi_{L}$, where

$$
\Phi_{L} \simeq \Omega^{1 / 5} d / L \text {. }
$$

Note that if $\Phi \lesssim \Phi_{L}$ the effective size of the chains along the P-nematic director, $R_{x},(\mathrm{Eq} .(6.8)$ ) is larger than the intrinsic coil thickness, $R_{\perp} \sim(L d / l) \Omega^{-2 / 5}$ (Eq. (6.1)). Thus a complete ordering of opaque discs $\left(\psi \sim \psi_{0} \simeq R_{\perp} / R_{\|}\right)$takes place at the concentration $\Phi_{L}$; at the same point the characteristic volume $V_{\psi}$, given by equation (6.6), coincides with the true volume of the coil, $R_{\|}^{2} R_{\perp}$, and the coils occupy nearly the whole space. For higher concentrations $\left(\Phi \gtrsim \Phi_{L}\right)$ we enter into the scaling region $P_{s}$, that we describe in terms of anisotropic blobs.

6.2.2. The Region $\mathrm{P}_{\mathrm{s}}$ : Melt of Anisotropic Blobs. - Above the concentration $\Phi_{L}$ equation (6.10), (in region $P_{s}$ ) the coils overlap strongly. Since each intersection between two coils costs a large energy $U \gtrsim 1$ (as the condition (6.9) is always fulfilled in the region $\mathrm{P}_{\mathrm{s}}$ ), the internal statistics of the chains is strongly disturbed by the interactions between chains. However, it is clear that short chain fragments must obey the isolated chain statistics given by equation (6.1). For the description of the solution in the $P_{s}$ region we propose the following modified scaling picture.

Let us consider the solution at some intermediate concentration inside the $\mathrm{P}_{s}$ region: $\Phi_{\mathrm{IP}} \lesssim \Phi \lesssim \Phi_{\mathrm{PS}}$ (see Fig. 4 ), where $\Phi_{\mathrm{IP}}$ is defined in equation (6.3), and

$$
\Phi_{\mathrm{PS}} \simeq \Phi_{5}=\Omega^{-1 / 5} \frac{d}{l}, \quad L>L_{5}
$$

$\left({ }^{6}\right)$ The same result is obtained by minimization of the free energy considered in paper II, (Eqs. (II.2.1-II.2.3)) 
The lowest chain length, $\Lambda$, in the $P_{s}$ region at a given $\Phi$ is given by equation $(6.10)$ :

$$
\Lambda \simeq d \Omega^{1 / 5} / \Phi,
$$

note that $\Lambda \gtrsim L_{5}$ as $\Phi \lesssim \Phi_{\mathrm{PS}}$. For the chains of length $\Lambda$ the orientation angle $\psi$ in the P-phase (Eq. (6.7)) is of the order of the self-orientation angle $\psi_{0}(\Lambda) \sim\left(\Lambda / l_{2}\right)^{1 / 2} \sim R_{\perp} / R_{\|} \sim$ $\Omega^{-2 / 5}\left[\frac{d^{3}}{l^{3} \Phi}\right]^{1 / 2}$ (see Eq. (6.1)). The coils (of length $\Lambda$ ) are closely packed; each of them "touches" only the neighboring chains. The energy of a contact (the interaction energy per chain) is of order unity.

Longer chains at a given volume concentration $\Phi$ can be cut into pieces (blobs) of contour length $\Lambda$, then the above results can be applied to these blobs. In particular, the internal statistics of the blobs is practically undisturbed by interactions with other blobs, and the statistics at scales larger than $\Lambda$ is strongly disturbed as the chains strongly overlap. The orientation angle of a blob in the P-phase, $R_{\perp} / R_{\|}$, coincides with the undisturbed self orientation angle, $\psi_{0}(\Lambda) \sim\left(\Lambda / l_{2}\right)^{1 / 2}$ (see Eqs. (6.1) and (I.2.5)), and also with the global orientation angle in the P-nematic phase, $\psi$. As the blob is longer than $L_{5}$, it can be viewed as a monisto of $\Lambda / L_{5}$ coins of volume $d R_{5}^{2}$, the statistics of the chain of coins being Gaussian in the $y$ and $z$ directions and completely stretched in the $x$ direction (this scaling picture has been described in Sect. I.3.3). Note that although an isolated chain of coins is completely stretched along the $x$-axis because of the excluded volume repulsion, in a semidilute solution the excluded volume interactions are screened at scales larger than $\Lambda$ (along a polymer chain); as a result the chain statistics at scales larger than the blob size are Gaussian. Thus the solution is characterized by the following parameters:

$$
\begin{gathered}
\Lambda \simeq d \Omega^{1 / 5} / \Phi ; \quad \psi \sim \psi_{0}(\Lambda) \sim \Omega^{-2 / 5}\left[\frac{d^{3}}{l^{3} \Phi}\right]^{1 / 2} ; \\
\xi_{y}=\xi_{z} \sim R_{\|}(\Lambda) \sim(d l / \Phi)^{1 / 2} \Omega^{1 / 5} ; \quad \xi_{x} \sim R_{\perp}(\Lambda) \sim\left(d^{2} / l\right) \Phi^{-1} \Omega^{-1 / 5} ; \\
R_{y}=R_{z} \sim \xi_{y}(L / \Lambda)^{1 / 2} \sim(L l)^{1 / 2} \Omega^{1 / 10} ; \quad R_{x} \sim \xi_{x}(L / \Lambda)^{1 / 2} \sim\left(d^{3} L / l^{2}\right)^{1 / 2} \Omega^{-3 / 10} \\
\text { (in region } \mathrm{P}_{\mathrm{s}} \text { ). }
\end{gathered}
$$

Note that $\Phi \sim\left(\Lambda d^{2}\right) /\left(\xi_{x} \xi_{y} \xi_{z}\right)$ : the solution can be considered as a melt of blobs, as in the isotropic region $\mathrm{I}_{\mathrm{s}}$. Note also that $\psi \sim \xi_{x} / \xi_{y} \sim\left(\Lambda / l_{2}\right)^{0.5}$ The radius $R_{y}$ coincides with the size of an isolated chain $R_{\mid l}(L)$ for $L \lesssim l_{2}$, and $\xi_{x} / \xi_{y} \sim R_{x} / R_{y} \sim\left(\Phi_{\mathrm{IP}} / \Phi\right)^{1 / 2} \ll 1$.

The typical orientation energy per blob is $\mathcal{F}_{\text {ord }} \sim \psi^{-2} \lambda / l_{2} \sim 1$ (cf. with the forth term in Eq. (2.2)), and the typical interaction energy is also of order unity: the blobs $\Lambda$ are simultaneously concentration and interaction blobs. Therefore, the total free energy of the $P_{s}$ phase is of order unity per blob $\left({ }^{7}\right)$.

At the concentration $\Phi_{\mathrm{IP}} \sim(d / l)^{3} \Omega^{-08}$ (Eq. (6.3)) $\Lambda$ approaches $l_{2}$, simultaneously $\psi \sim 1$ (see Eq. (6.13)) and the melt of anisotropic blobs transforms into a melt of isotropic blobs, $\mathrm{I}_{\mathrm{s}}$. The corresponding first order transition occurs in the region between $\Phi_{I}$ and $\Phi_{P}, \Phi_{I} \sim \Phi_{P} \sim$ $\Phi_{\mathrm{IP}}$ where $\Phi_{\mathrm{IP}}$ is given by equation (6.5).

$\left({ }^{7}\right)$ For an isotropic system the condition that the blobs tightly fill the space without much overlapping defines the blobs unambiguously. This is not the case for a nematic system. One can define smaller blobs, and simultaneously set larger orientation angle $\psi$, so that the blobs would still fill the volume of the system tightly. However with these smaller blobs the total free energy, which is proportional to the number of blobs, is larger. Therefore smaller blobs are thermodynamically unfavorable. 
At the concentration $\Phi_{\mathrm{PS}}(6.11), \Lambda$ approaches $L_{5}$. The character of the blobs changes from anisotropic (disc-like) "thick blobs" (regime $\mathrm{P}_{\mathrm{s}}$ ) to the regime of "flat blobs", where the blob size $\xi_{x}$ is of the order of the chain diameter $d$. It turns out that this transition is accompanied by an additional smectic ordering: it is actually a second order transition to a $\mathrm{S}_{\mathrm{A}}$-smectic phase which is considered below.

6.2.3. The $\mathrm{P}_{\mathrm{L}}$ and $\mathrm{P}_{\text {ct }}$ Phases. - Let us now consider the vicinity of the $\Phi_{* *}$ line in the interval $l \lesssim L \lesssim L_{6}$, where a transition from the isotropic to the $\mathrm{P}_{\mathrm{L}}$-phase takes place (see Eq. (6.5)). The orientation angle in this phase is given by the first line of equation (4.5): it is nearly independent of both $l$ and $l_{2}$. The correlation corrections are small near the transition line: $\mathcal{N} \ll 1$ (see Eq. (2.5), with $\psi \sim 1$ ).

The free energy of the system is determined by equations (2.1, 2.5). As the concentration increases above $\Phi_{\mathrm{IP}} \sim \Phi_{* *}$, the correlation corrections become more important giving rise to a new free energy minimum at $\psi \ll 1$ corresponding either to the $\mathbf{P}_{\text {opq }}$ phase considered above (for $L_{7}<L<L_{6}$ ), or to the new correlation phase $\mathrm{P}_{\text {ct }}$ (for $l<L<L_{7}$ ). Here $L_{6}=l_{2} \Omega^{-2 / 5}$, $L_{7}=l \Omega^{3 / 5} ; L_{7} / L_{6}=(d / l)^{2} \ll 1$. The characteristics of the first order phase transitions $\mathrm{P}_{\mathrm{L}} \rightarrow \mathrm{P}_{\mathrm{opq}}$, and $\mathrm{P}_{\mathrm{L}} \rightarrow \mathrm{P}_{\mathrm{ct}}$ are:

$$
\begin{gathered}
\Phi\left(\mathrm{P}_{\mathrm{L}}\right) \sim \frac{d}{L} \ln \frac{L_{6}}{L} ; \quad \Phi\left(\mathrm{P}_{\mathrm{opq}}\right) \sim \frac{d}{L}\left(\ln \frac{L_{6}}{L}\right)^{2} ; \quad \psi\left(\mathrm{P}_{\mathrm{opq}}\right) \sim\left(\frac{L}{L_{6}}\right)^{1 / 2} /\left(\ln \frac{L_{6}}{L}\right)^{2} \\
\Phi\left(\mathrm{P}_{\mathrm{L}}\right) \sim \frac{d}{L} \ln \frac{l}{d} ; \quad \Phi\left(\mathrm{P}_{\mathrm{ct}}\right) \sim \frac{d}{L}\left(\ln \frac{l}{d}\right)^{2} ; \quad \psi\left(\mathrm{P}_{\mathrm{ct}}\right) \sim \frac{d}{l} /\left(\ln \frac{l}{d}\right)^{3}
\end{gathered}
$$

where $\psi\left(\mathrm{P}_{\mathrm{opq}}\right)$ and $\psi\left(\mathrm{P}_{\mathrm{ct}}\right)$ are the orientation angles in the corresponding phases at the transition line.

The orientation angle inside the $P_{c t}$ region is determined by minimization of the free energy, equation (2.1); it is always smaller than $d / l$ :

$$
\psi \sim \frac{d}{l}\left(\frac{d}{\Phi L}\right)^{3 / 2}
$$

The $\mathrm{P}_{\mathrm{ct}}$ state is characterized by unique properties: here the interaction energy between two polymer chains is small, $U \ll 1$, but the typical number of contacts between two intersecting chains is large, $\mathcal{N} \sim\left(\frac{d}{l \psi}\right)^{2 / 3} \gg 1$. On the other hand the total interaction energy per chain, $U_{\text {int }} \sim 1$, so that the interaction blob length coincides with the chain length: $\Lambda \sim \frac{d \mathcal{N}}{\Phi} \sim L$. Therefore the correlation lengths in this phase coincide with the chain sizes. The coil size in the $y-z$ (PMF) plane is the same as for an isolated coil:

$$
R_{z}=R_{y}=R_{\|}
$$

where $R_{\|}$is given by equation (6.1). The effective size in the $x$-direction is

$$
R_{x} \sim \psi R_{\|}
$$

(one can directly check that $\psi R_{x}$ is larger than the self-thickness of a polymer coil, $R_{\perp}$ ). The number $\tilde{n}$ of chains overlapping with a given chain is:

$$
\tilde{n} \sim c R_{\|}^{3} \psi=\frac{\Phi}{L d^{2}} R_{\|}^{3} \psi .
$$

